\title{
Code KARAT in simulations of power microwave sources including Cherenkov plasma devices, vircators, orotron, E-field sensor, calorimeter etc.
}

\author{
V.P. Tarakanov ${ }^{1,2}$ \\ ${ }^{1}$ JIHT RAS, Moscow, Russia, karat@tarak.msk \\ ${ }^{2}$ National Res.Nuclear Univ.MEPhI, , Moscow, Russia
}

This paper deals with some last simulations made by electromagnetic PiC code KARAT[1] that is aimed at the solution of $1,2 \& 3 \mathrm{D}$ non-stationary electrodynamics problems in a complicated geometry involving beams, plasmas, dielectrics, ionization etc.

\section{A conical vircator}

The vircator with conical electrodes for ballistic focusing of the electron beam was offered and investigated [2] including the dynamics of electrons in vircator and the dynamics of the $\mathrm{VC}$ as a whole.

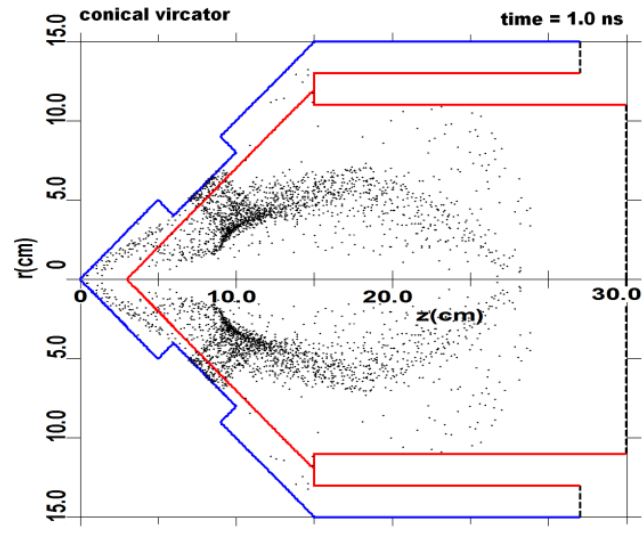

Fig. 1. The evolution of the electron beam and the virtual cathode (VC) in a conical vircator

The VC has the form of curvilinear figure of rotation. It oscillates in both radial and axial directions. The middle VC position depends on the beam current. The generation is resonant at a single frequency, depending on the beam current

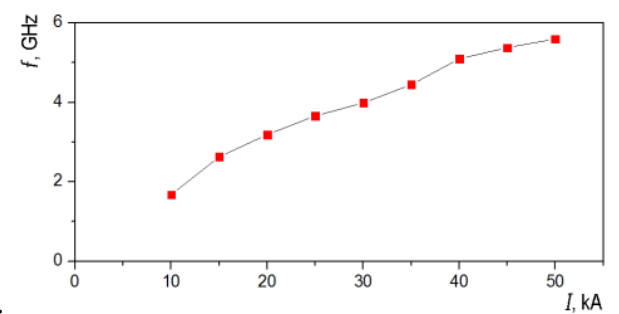

Fig. 2. The dependence of the resonance frequency of the conical vircator generation of the beam current

At the beam current from the range of $10-50 \mathrm{kA}$ the oscillation frequency increases monotonically from 2 to $6 \mathrm{GHz}$ with the beam current increase

\section{Efficient relativistic orotron with mode selection}

A high-power $\mathrm{V}$-band relativistic orotron on a $\mathrm{TM}_{03}$ oscillation mode has an ovesized $(\mathrm{D} / \mathrm{k} \approx 2.7)$ electrodynamic structure and diffractive output in the $\mathrm{TM} 02$ mode [3]. Single-mode operation of the oscillator is en- sured by the cyclotron selection of the working axisymmetric mode, with simultaneous depression of competing non-axisymmetric modes by means of cutting longitudinal slits in the wall of the SWS. The output power is 350 MW with a power conversion efficiency of $31 \%$ when using a $3.6 \mathrm{kA}, 310 \mathrm{keV}$ electron beam transported in the $3.9 \mathrm{~T}$ magnetic field.

The modeling demonstrated that the simultaneous application of cyclotron and electrodynamic methods of mode selection in the orotron ensures the single-mode operation of the oscillator. The simulated microwave efficiency of the device is close to the theoretical limit. Furthermore, the orotron retains its efficiency $30 \%$ even in the case when the energy of the driving electron beam is reduced to $200 \mathrm{keV}$. Therefore, it seems to be possible to build a compact pulsed source of millimeter-wave radiation with a power of about $10^{8} \mathrm{~W}$, based on an orotron and a compact high-current electron accelerator.

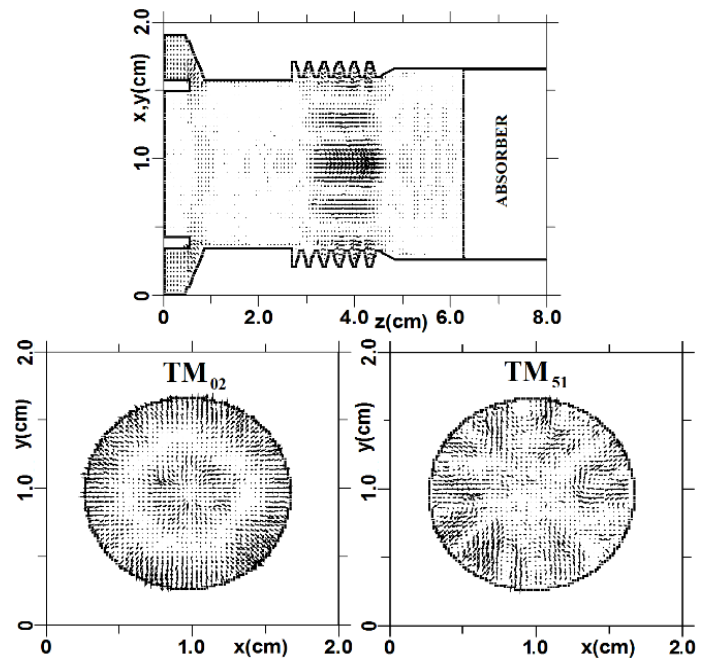

Fig. 3. Snapshots of electric field vectors in 3D modeling: in the axial middle plane of the orotron (top), and in a cross section of the output waveguide: in the desired operation regime (left) and in the regime with mode competition (right)

3D simulations of the orotron employed the Cartesian version of code. In these runs, the grid division was typically $120 \times 120 \times 450$ and the number of macro particles was about 600000 . The primary goal of the modeling was to observe the composition of competing modes. It is possible to reduce the $Q$-factor of a non-symmetric mode by means of cutting longitudinal slits through the wall of the waveguide (interrupting the azimuthal currents). The number of slits should not equate the azimuthal index of the mode desired to suppress. Indeed, introduction of nine slits in the SWS wall in 3D modeling notably expanded the band of magnetic fields, where $\mathrm{TM}_{03}$ oscillation dominates in the orotron. 


\section{Simulation of wide-aperture liquid calorimeters}

Time domain simulation of diffraction and absorption effects in diagnostics of short high-power microwave pulses using wide-aperture calorimeters [4] was made. To take into account the frequency dispersion of the absorbing mixture, a Lorentz model implemented in the code was applied, based on the presentation of current in the medium by harmonic oscillators.

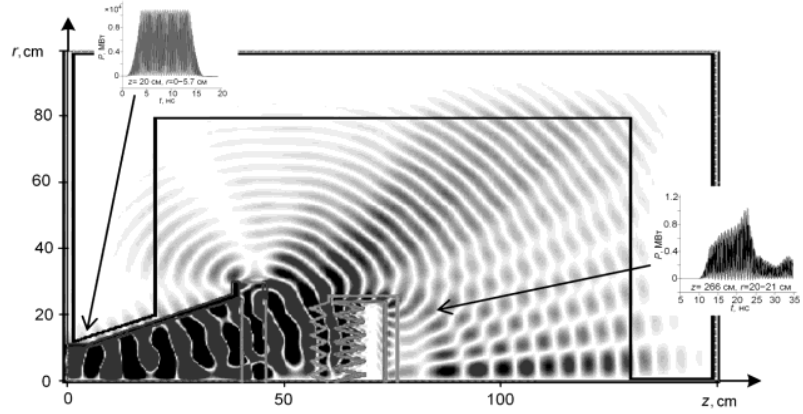

Fig. 4. Simulated $B_{\text {theta }}$ field pattern with a fundamental frequency of $3.8 \mathrm{GHz}$ in the vicinity of radiating horn and the load of the calorimeter. Insets show the waveforms of power flux in the neck of the horn and behind the device

The simulation demonstrated that, first, a diffraction field is superimposed on the wave field, which passes through the device with attenuation, while the interference pattern at the front and the decay of the RF pulse is nonstationary. In the field passing through the load, lowfrequency components begin to prevail, due to a stronger decay of the high frequency.

\section{UWB strip-line sensor antenna}

In designing of the compact semiconductor high power pulse radiator the 3D simulations of the radiator and the compact strip-line sensors were performed [5]. The e/m pulse with the rise time of $50 \mathrm{ps}$ was realized by TEM-horn antennas. The structure of elm field was measured by various sensors including the new KARAT designed E-field sensor non-symmetrical two-wire type with dielectric stalk with high $\varepsilon=16$ and small crosssection and $40 \mathrm{~cm}$ length.

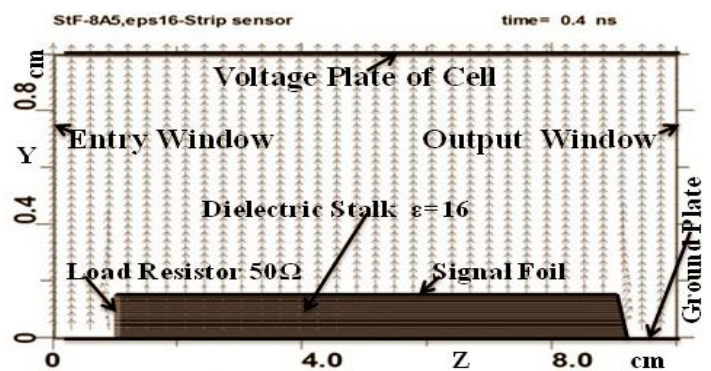

Fig. 5. Sensor model of $L_{8 C S}=8 \mathrm{~cm}$ length (YZ-section along $x=0.8 \mathrm{~cm})$ in the gauge cell with $1 \mathrm{~cm}$ gap. Vertical arrows are $\boldsymbol{E}_{0}(t)$ - electric field vectors at $t=0.4 \mathrm{~ns}$ moment

Simulations of the sensor model gives values by closed to experimental values.

\section{High current relativistic gyrotron}

A compact relativistic gyrotron, operating at $3-\mathrm{mm}$ wavelength band was realized with $250 \mathrm{kV}, 100 \mathrm{~A}, 1 \mu \mathrm{s}$ helical electron beam, forming by a thermionic magnetron-injection electron gun. The device operated in the rotating $\mathrm{TE}_{12,5,1}$ mode of a circular waveguide cavity. The mode was transformed into a Gaussian wave beam by a built-in mode converter.
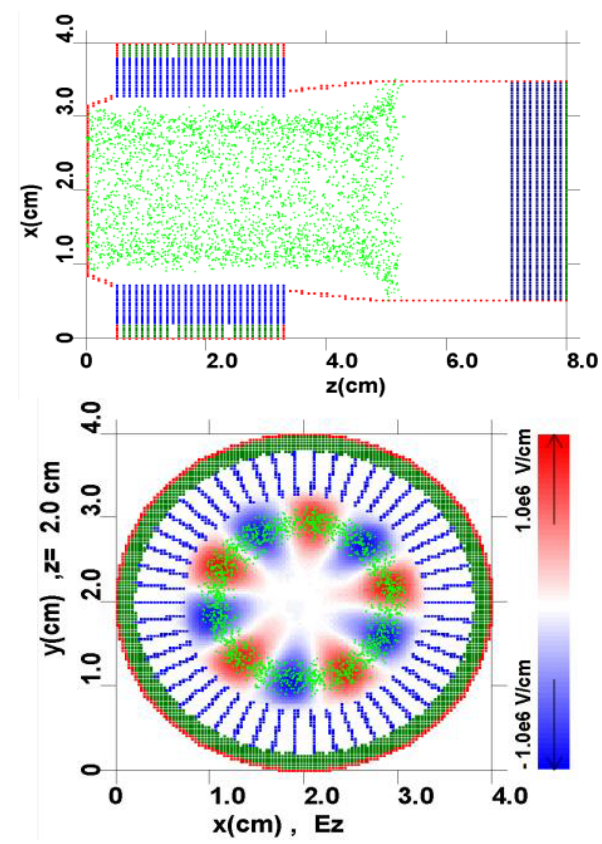

Fig. 6. The gyrotron construction, electron beam and $\mathrm{Ez}$ of generated field

\section{Evolution of plasma in plasma electronics devices}

Until this work [6] in theoretical investigations parameters of plasmas inside a plasma electronics device were considered constant. We simulated experimental setup were the duration of beam pulse was more hundred of nsec. PiC simulation shows that under conditions when beam current $\sim 2 \mathrm{kA}$, energy $\sim 500 \mathrm{keV}$, guiding magnetic field $\sim 4 \mathrm{kG}$, initial Xe plasma density $\sim 10^{12} \mathrm{~cm}^{-3}$ in regime when output microwave power ordered some hundreds $\mathrm{kW}$ to time hundred ns kinetic energy of plasma electrons achieves hundred $\mathrm{keV}$. As electrons are frozen in guiding magnetic field then they leave an inner volume alongside of magnetic field and are absorbed on left and right boundaries. A positive potential ordered hundred Volt appears. As plasma ions are not frozen they moves to a side wall and plasma density reduces. As result an optimal parameters of system for amplification or generation of $\mathrm{e} / \mathrm{m}$ wave are violated and output pulse breaks off.

Work is supported by RAS program "Elemental base and power semiconducting electronics devices" (ОЭ-8, acad. I.V. Grekhov).

\section{References}

1. V.P. Tarakanov. User's Manual for Code KARAT, BRA Inc., Va., USA, (1992.)

2. A.E. Dubinov, V.P. Tarakanov IEEE TRANSACTIONS ON PLASMA SCIENCE, V.44, NO. 8, 2016.

3. E.M. Totmeninov, I.V. Pegel, and V.P. Tarakanov Physics of Plasmas 24, 053106 (2017).

4. V.P. Tarakanov, A.I. Klimov, I.V. Pegel (in press).

5. V. Fedorov (in press).

6. P.S. Strelkov, V.P. Tarakanov (in press). 